Vietnam Journal of Mechanics, VAST, Vol. 33, No. 4 (2011), pp. $203-213$

\title{
FINITE ELEMENT SIMULATION OF STRAIN LOCALIZATION IN INELASTIC SOLIDS BY ENERGY RELAXATION
}

\author{
B. T. Trinh, K. Hackl \\ Ruhr-Universität Bochum, Universitätsstr. 150 \\ D-44801 Bochum, Germany
}

\begin{abstract}
A new approach for the treatment of strain localization in inelastic material is proposed. It is based on energy minimization principles associated with micro-structure developments. Shear bands are treated as micro-shearing of rank-one laminates. It is assumed that the thickness of the shear band represented by its volume fraction tends to zero, and the energy inside the shear band is a function of the norm of the strain field. The existence of shear bands in the structure leads to an ill-posed problem which can be solved by means of energy relaxation. The performance of the proposed concept is demonstrated through numerical simulation of tension test under plane strain conditions. Numerical results show that mesh sensitivity can be completely removed.
\end{abstract}

Keywords: Energy relaxation, shear band, strain localization.

\section{INTRODUCTION}

Regions of high strain localization by intense shearing are referred to as shear bands. The formation of shear bands is accompanied by a softening response, characterized by a decrease in strength of the material with accumulated inelastic strain, often leading to complete failure $[1,2]$. Predictions of the onset and evolution of shear bands play an important role in determining the safety of structures, improving mechanical properties of material and designing material microstructure.

In recent years a new methodology based on energy relaxation has been developed to simulate the development of material microstructures [3]-[9]. The advantage of this theory, when applied to the problem at hand, is the natural formation of shear bands based on the energy minimization principles associated with micro-structure developments.

In this paper a theoretical framework for the treatment of shear localizations in inelastic materials at small strains is developed. The theory is based on energy minimization principles associated with micro-structure developments under the assumption of a shear band of zero thickness and of a special form of the energy within the shear band. Shear bands are treated as laminates of first order. The problem of the non-convex energy arising due to the formation of shear bands is solved by energy relaxation in order to ensure 
that the corresponding problem is well-posed. Numerical simulations of a tension test are shown in order to evaluate the performance of the proposed concept.

\section{SHEAR BANDS AS SPECIAL LAMINATES}

In this paper localization phenomena are regarded as micro-structure developments associated with nonconvex potentials. We assume the micro-structures to consist of two domains: a low-strain domain and a high-strain one. Let us consider a so-called RVE (representative volume element) obtained by zooming in on the region around the point $A$ as shown in Fig. 1. The RVE is split into two volume fractions: the volume fraction $\xi$ of the low-strain domain and the volume fraction $(1-\xi)$ of the high-strain domain.

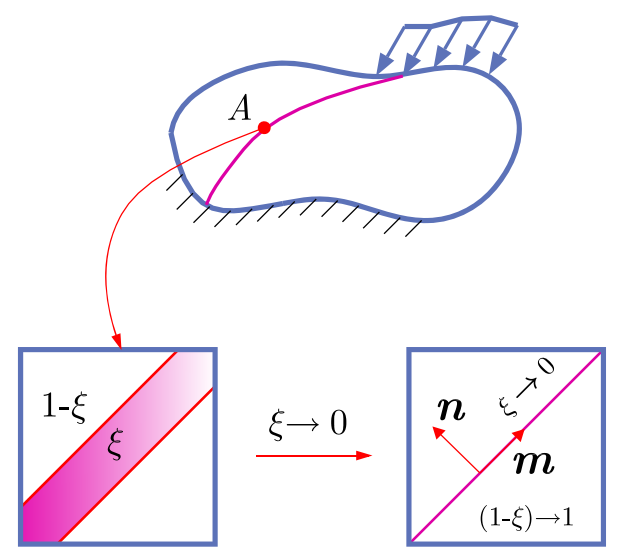

Fig. 1. Shear band is treated as the micro-shearing of a rank-one laminate

When strain localization occurs, the following assumptions related to the volume fraction $\xi$ are made:

(1) After the onset of localization, the width of the shear band represented by the volume fraction $\xi$ tends to zero

$$
\xi \rightarrow 0 .
$$

(2) Most of the deformation is concentrated parallel to the band

$$
\boldsymbol{m} . \boldsymbol{n}=0
$$

where $\boldsymbol{m}$ and $\boldsymbol{n}$ are two unit vectors giving the direction of shear band evolution.

(3) The potential (energy) inside the shear band $W_{2}$ is assumed to satisfy

$$
W_{2}(\xi \varepsilon)=|\xi| W_{2}(\varepsilon) .
$$

For example $W_{2}(\varepsilon)$ may be taken in the following form

$$
W_{2}(\varepsilon)=A\|\epsilon\|
$$

where $A$ is a material parameter.

The first assumption is based on the observations that the width of shear bands is normally very small relative to the overall dimension of the system under consideration 
$[10,11]$. This assumption corresponds to the concept of strong discontinuities proposed in $[12,13]$ among others.

The second assumption based on the results of the experiment by Finno et al, 1996 [14]. In their experiment, they showed that the normal movements are much smaller than those in the tangent direction inside the shear band. This assumption implies the two vectors $\boldsymbol{m}$ and $\boldsymbol{n}$ being orthogonal.

According to the third assumption, the potential inside a shear band is positive homogeneous of first-order in the strain field (3). We will see later on, that only for this very form of the potential as given in eq. (4), it has the desired property leading to strong discontinuities.

In the following considerations we develop a simple approach to the treatment of shear localization based on these assumptions.

\section{TWO-DIMENSIONAL PROBLEM}

\subsection{Micro-strain}

In the two-dimensional problem the micro-strains $\varepsilon_{1}$ and $\varepsilon_{2}$ can be written as

$$
\begin{gathered}
\varepsilon_{1}=\boldsymbol{\varepsilon}-\xi(\boldsymbol{a} \otimes \boldsymbol{n})^{\mathrm{s}} \\
\boldsymbol{\varepsilon}_{2}=\boldsymbol{\varepsilon}+(1-\xi)(\boldsymbol{a} \otimes \boldsymbol{n})^{\mathrm{s}}=\boldsymbol{\varepsilon}-\xi(\boldsymbol{a} \otimes \boldsymbol{n})^{\mathrm{s}}+(\boldsymbol{a} \otimes \boldsymbol{n})^{\mathrm{s}}
\end{gathered}
$$

where $(\boldsymbol{a} \otimes \boldsymbol{n})^{\mathrm{s}}=\frac{1}{2}(\boldsymbol{a} \otimes \boldsymbol{n}+\boldsymbol{n} \otimes \boldsymbol{a})$.

Let us define $s$ by

$$
\xi \boldsymbol{a}=s \boldsymbol{m},
$$

where $\|\boldsymbol{m}\|=1$. Herein $\boldsymbol{m}$ and $\boldsymbol{n}$ are two unit vectors giving the direction of shear band evolution, $s$ is an appropriately rescaled variable.

On inserting eq. (7) into eqs. (5) and (6), we have

$$
\begin{gathered}
\boldsymbol{\varepsilon}_{1}=\boldsymbol{\varepsilon}-s(\boldsymbol{m} \otimes \boldsymbol{n})^{\mathrm{s}}, \\
\varepsilon_{2}=\boldsymbol{\varepsilon}-s(\boldsymbol{m} \otimes \boldsymbol{n})^{\mathrm{s}}+\frac{s}{\xi}(\boldsymbol{m} \otimes \boldsymbol{n})^{\mathrm{s}} .
\end{gathered}
$$

As $\xi$ tends to zero $\frac{s}{\xi}$ will grow out of bounds. Thus, eq. (9) can be simplified as

$$
\boldsymbol{\varepsilon}_{2} \approx \frac{s}{\xi}(\boldsymbol{m} \otimes \boldsymbol{n})^{\mathrm{s}}
$$

\subsection{Relaxed potential}

The mixed potential of the two domains can be written in the following form

$$
W^{m i x}(\varepsilon)=W_{1}\left(\varepsilon-s(\boldsymbol{m} \otimes \boldsymbol{n})^{\mathrm{s}}\right)+|s| W_{2}\left((\boldsymbol{m} \otimes \boldsymbol{n})^{\mathrm{s}}\right) .
$$

The relaxed potential is computed in order to ensure the well-posedness of the boundary-value problem governing the emergence of a shear band. It is obtained by the minimization procedure

$$
W_{R}(\varepsilon)=\inf \left\{W^{m i x}(\varepsilon) \mid s, \boldsymbol{m}, \boldsymbol{n}:\|\boldsymbol{m}\|=\|\boldsymbol{n}\|=1\right\} .
$$


Table 1. Minimization problem: $\inf W(s)$

\begin{tabular}{|ll|}
\hline Scalar minimization problem: & $\inf _{s} W(s)$ \\
Potential: & $W(s)=a s^{2}+b s+c|s|$ with $c>0, a>0$ \\
Solution: & $\inf _{s} W(s)=-\frac{1}{4 a}(|b|-c)_{+}^{2}$ \\
Minimizer: & $s=-\frac{1}{2 a}(|b|-c)_{+} \operatorname{sign}(b)$ \\
Abbreviations: & $(|b|-c)_{+}= \begin{cases}0 & \text { for }|b| \leq c \\
|b|-c & \text { for }|b|>c\end{cases}$ \\
& $\operatorname{sign}(b)=\frac{|b|}{b}$ for $b \neq 0$ \\
\end{tabular}

Let us consider two specific potentials representing the behaviour at small and large strains, respectively

$$
\begin{gathered}
W_{1}(\varepsilon)=\frac{1}{2} \varepsilon: \mathcal{C}: \varepsilon, \\
W_{2}(\gamma)=A\|\gamma\|,
\end{gathered}
$$

where $\mathcal{C}$ is symmetric fourth-order, positive definite tensor. Substituting (13) and (14) into (11), one gets

$$
W^{m i x}(\varepsilon)=\frac{1}{2}(\varepsilon-s \gamma): \mathcal{C}:(\varepsilon-s \gamma)+A|s|\|\gamma\|
$$

where $\boldsymbol{\gamma}=(\boldsymbol{m} \otimes \boldsymbol{n})^{\mathrm{s}}$.

On inserting (2) into (15) we have the simplified form of the mixed energy

$$
W^{m i x}(\varepsilon)=\frac{1}{2}(\varepsilon-s \gamma): \mathcal{C}:(\varepsilon-s \gamma)+\frac{A}{\sqrt{2}}|s| .
$$

Using the results in Table 1, minimization of (16) with respect to $s$ yields

$$
s=\frac{\operatorname{sign}(\varepsilon: \mathcal{C}: \gamma)}{(\gamma: \mathcal{C}: \gamma)}\left[|\varepsilon: \mathcal{C}: \gamma|-\frac{A}{\sqrt{2}}\right]_{+},
$$

and the potential (16) with $s$ given by (17) takes the form

$$
\inf _{s} W^{m i x}(\varepsilon)=\frac{1}{2} \varepsilon: \mathcal{C}: \varepsilon-\frac{1}{2} \frac{\left[|\varepsilon: \mathcal{C}: \gamma|-\frac{A}{\sqrt{2}}\right]_{+}^{2}}{\gamma: \mathcal{C}: \gamma} .
$$

Herein $a=\frac{1}{2} \gamma: \mathcal{C}: \boldsymbol{\gamma}, b=-\varepsilon: \mathcal{C}: \boldsymbol{\gamma}, c=\frac{A}{\sqrt{2}}$. Easily one can recognise that $a$ is positive due to the positive definiteness of the fourth-order tensor $\mathcal{C}$. 


\subsection{Computation of stress and the tangent operator}

The stress and the tangent operator are derived from the direct derivative of the relaxed potential (12). The derivative of (12) reads

$$
\frac{\partial W_{R}}{\partial \boldsymbol{\varepsilon}}=\frac{\partial W^{m i x}}{\partial \boldsymbol{\varepsilon}}+\frac{\partial W^{m i x}}{\partial s} \frac{\partial s}{\partial \boldsymbol{\varepsilon}}+\frac{\partial W^{m i x}}{\partial \boldsymbol{m}} \frac{\partial \boldsymbol{m}}{\partial \boldsymbol{\varepsilon}}+\frac{\partial W^{m i x}}{\partial \boldsymbol{n}} \frac{\partial \boldsymbol{n}}{\partial \boldsymbol{\varepsilon}} .
$$

It is observed that the three last terms in eq. (19) vanish due to the stationarity condition of the minimization problem (12). Thus, the relaxed stress which is an appropriate average of the two micro-stresses has the form

$$
\boldsymbol{\sigma}=\frac{\partial W^{m i x}}{\partial \varepsilon}
$$

Considering the form of the potential (18) we obtain

$$
\boldsymbol{\sigma}=\mathcal{C}: \varepsilon-s \mathcal{C}: \gamma
$$

The tangent operator is given by

$$
\mathcal{A}=\frac{\partial^{2} W_{R}}{\partial \varepsilon^{2}}=\frac{\partial \boldsymbol{\sigma}}{\partial \varepsilon}=\mathcal{C}-(\mathcal{C}: \gamma) \otimes \frac{\partial s}{\partial \varepsilon}-s \frac{\partial(\mathcal{C}: \gamma)}{\partial \varepsilon}
$$

\subsection{Localization criterion}

In the derivations above a central role is played by the quantity

$$
L=\left[|\varepsilon: \mathcal{C}: \gamma|-\frac{A}{\sqrt{2}}\right] .
$$

As the process of deformation progresses, $L$ may be negative, zero or positive. A positive value in turn signals the onset of localization. A criterion that can be shown to be equivalent to the well-known notion of loss of ellipticity.

i. $L \leq 0$ : we have $s=0$. The relaxed potential $W_{R}(\varepsilon)$ reduces to the elastic strain energy $\overline{W_{1}(\varepsilon)}$.

ii. $L>0$ : we have $s \neq 0$. A shear band starts to develop. The homogeneous deformation $\varepsilon$ decomposes into the two micro-strains $\varepsilon_{1}$ and $\varepsilon_{2}$. The nonconvex potential energy $W^{m i x}$ is replaced with the approximated rank-one convexification $W_{R}(\varepsilon)$ to ensure well-posedness of the problem.

\section{APPLICATION OF RELAXATION THEORY TO LINEAR ISOTROPIC MATERIAL}

The fourth-order isotropic elastic tensor $\mathcal{C}$ takes the form

$$
\mathcal{C}_{i j k l}=\lambda \delta_{i j} \delta_{k l}+\mu\left(\delta_{i k} \delta_{j l}+\delta_{i l} \delta_{j k}\right)
$$

or in the tensor notation

$$
\mathcal{C}=\lambda \boldsymbol{I} \otimes \boldsymbol{I}+\mu(\boldsymbol{I}+\overline{\mathcal{I}})
$$


where $\lambda$ and $\mu$ are Lamé constants.

The simplified form of the mixed energy is obtained by substituting (24) into eq. (16)

$$
W^{m i x}(\boldsymbol{\epsilon})=\frac{\lambda}{2}(\operatorname{tr} \boldsymbol{\epsilon})^{2}+\mu\left(\|\boldsymbol{\epsilon}\|^{2}-2 s \boldsymbol{m} \cdot \boldsymbol{\epsilon} \boldsymbol{n}+\frac{s^{2}}{2}\right)+\frac{A}{\sqrt{2}}|s| .
$$

Based on the minimization procedure the relaxed potential (12) reads

$$
W_{R}=\inf _{s, \boldsymbol{m}, \boldsymbol{n}} W^{m i x}=\frac{\lambda}{2} \operatorname{tr}(\boldsymbol{\epsilon})^{2}+\mu\|\boldsymbol{\epsilon}\|^{2}-2 \mu\left[\left|\epsilon_{m n}\right|-\frac{\alpha}{2}\right]_{+}^{2},
$$

where $\alpha=\frac{A}{\sqrt{2} \mu},\left|\epsilon_{m n}\right|$ is the maximum shear strain.

Solution $s$ yields

$$
s=\left(2\left|\epsilon_{m n}\right|-\alpha\right)_{+} \operatorname{sign}\left(\epsilon_{m n}\right)= \begin{cases}0 & \text { if }\left|\epsilon_{m n}\right|<\alpha / 2 \\ \left(2\left|\epsilon_{m n}\right|-\alpha\right) \operatorname{sign}\left(\epsilon_{m n}\right) & \text { if }\left|\epsilon_{m n}\right| \geq \alpha / 2\end{cases}
$$

The relaxed stress is obtained from eqs. (21) and (27)

$$
\boldsymbol{\sigma}=\lambda t r \boldsymbol{\epsilon}+2 \mu \boldsymbol{\epsilon}-2 \mu s(\boldsymbol{m} \otimes \boldsymbol{n})^{s} .
$$

On inserting (27) into (22) we get the tangent operator

$$
\mathcal{A}=\lambda \boldsymbol{I} \otimes \boldsymbol{I}+\mu(\mathcal{I}+\overline{\mathcal{I}})-2 \mu\left[(\boldsymbol{m} \otimes \boldsymbol{n})^{s} \otimes \frac{\partial s}{\partial \boldsymbol{\epsilon}}+s \frac{\partial(\boldsymbol{m} \otimes \boldsymbol{n})^{s}}{\partial \boldsymbol{\epsilon}}\right] .
$$

The capability of the proposed model is demonstrated through numerical simulation of a tension test in the next Section.

\section{TENSION TEST}

In this section the model presented in Section 4 are implemented into the finite element code FEAP [15]. In what follows we investigate a tension test under plane strain conditions. Different kinds of elements are used in this example as summarized in Fig. 2.

\begin{tabular}{clcl}
\hline Element type & Method & Number of Gauss points & Notation \\
\hline & MES $^{1}$ & $2 \times 2$ & MES \\
& EAS $^{2}$ & $2 \times 2$ & EAS \\
& QM6 $^{3}$ & $2 \times 2$ & QM6 \\
& Disp $^{4}$ & $2 \times 2$ & Q4 \\
\hline
\end{tabular}

Fig. 2. Notation of element type: $\mathrm{MES}^{1}$ - the mixed enhanced strain method [16], $\mathrm{EAS}^{2}$ - the enhanced assumed strain method [17], QM6 ${ }^{3}$ - the method of incompatible mode [18], Disp ${ }^{4}$ - the displacement method [19]

A sample is subjected to a prescribed vertical displacement under plane strain conditions. The geometry of the specimen and the boundary conditions imposed on the displacements as well as the material parameters are given in Fig. 3. In order to trigger the shear band formation a geometrical imperfection along the height of specimen is introduced. 


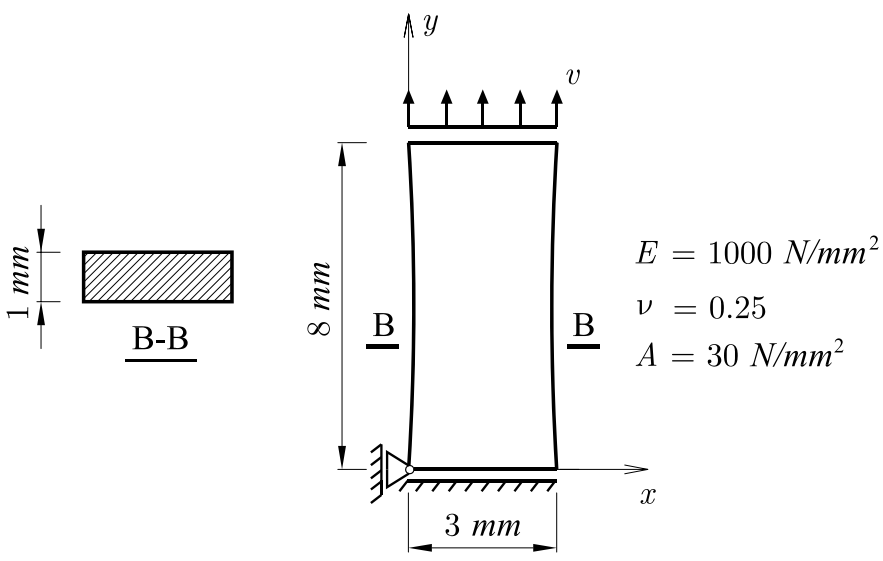

Fig. 3. Localization in tension. Geometry and boundary conditions

Four discretizations of the domain are considered: $3 \times 8,7 \times 18,14 \times 36,21 \times 54$ elements. The load-displacement curves behave identically for $v<0.3126 \mathrm{~mm}$ as shown in Figs. $4 \mathrm{a}$ and $5 \mathrm{a}$. The localization is delayed until the maximum shear strain is equal to $\frac{\alpha}{2}$ corresponding to $v \approx 0.3126 \mathrm{~mm}$, then the performance of diverse finite element methods starts to differ. The localization band is straight, at $45^{\circ}$ with the direction of principal strain.

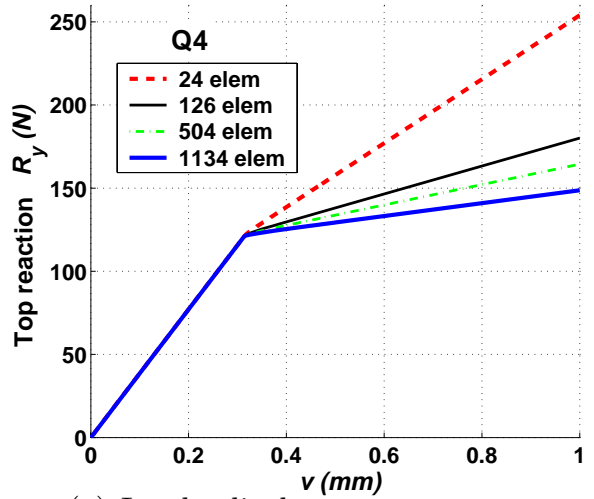

(a) Load - displacement response

\section{Relaxed energy $\left(\mathrm{Nmm} / \mathrm{mm}^{3}\right)$}
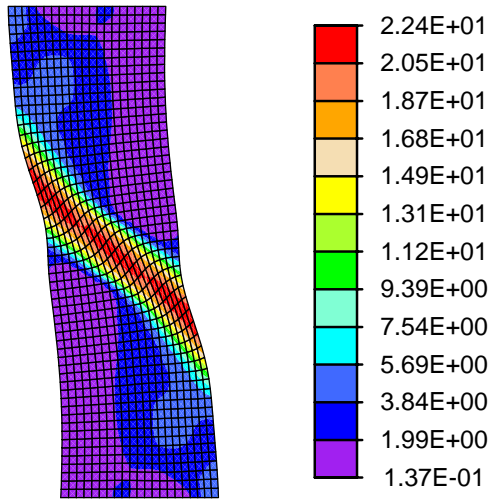

(b) Distribution of relaxed energy at $v=1.0 \mathrm{~mm}$

Fig. 4. Localization in tension by use of Q4 element (the displacement method)

The displacement method is unable to capture the localization and shows hardening behaviour as depicted in Fig. $4 \mathrm{a}$ and $4 \mathrm{~b}$. 


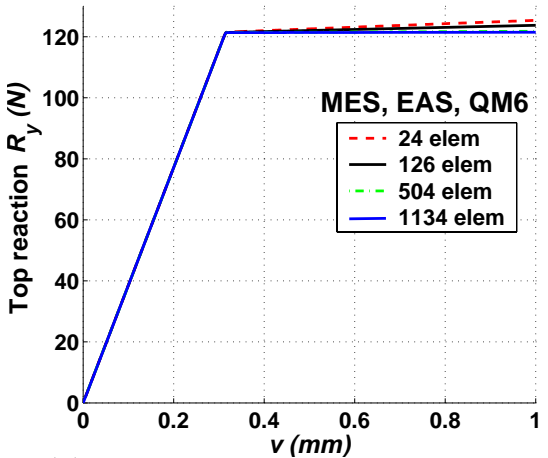

(a) Load - displacement response
Relaxed energy $\left(\mathrm{Nmm} / \mathrm{mm}^{3}\right)$
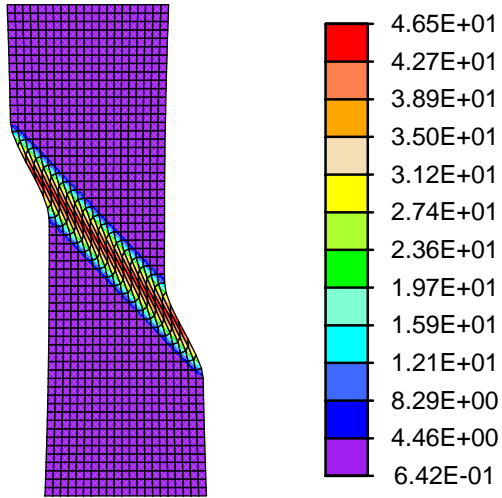

(b) Distribution of relaxed energy at $v=1.0 \mathrm{~mm}$

Fig. 5. Localization in tension by use of MES element (the mixed enhanced strain method), EAS element (the enhanced assumed strain method) and QM6 element (the incompatible mode method)

As we expected in Fig. 5a, the solutions obtained by using MES, EAS and QM6 elements are practically coincident in term of vertical reactions, thus showing mesh independence of the proposed relaxed theory. The distribution of the relaxed energy shown in Fig. 5b points out the localized elements.

The behaviour of the relaxed potential as well as the relaxed vertical stress $\sigma_{y}$ of the element 356 at the first gauss point inside the shear band is depicted in Fig. 6. After the

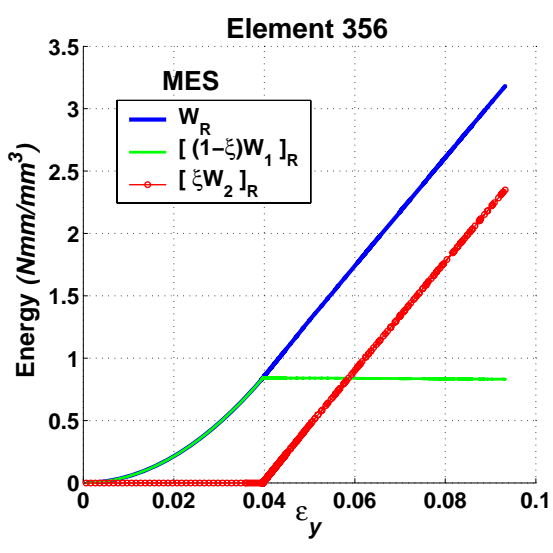

(a)

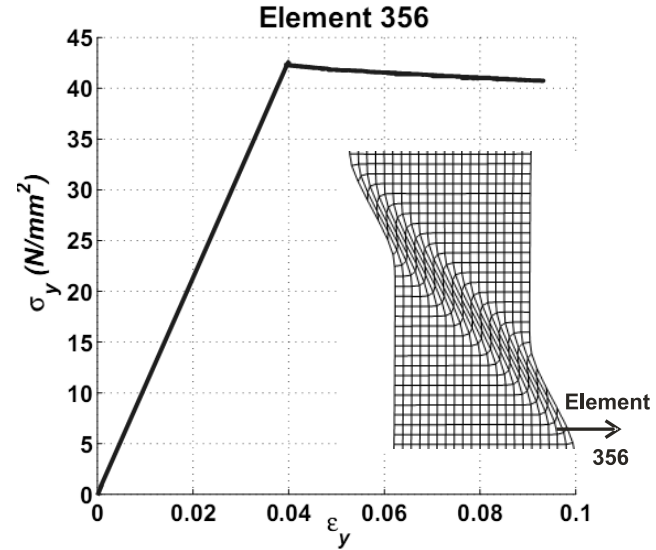

(b)

Fig. 6. Relaxed energy and relaxed normal stress $\sigma_{y}$ by use of MES element (the mixed enhanced strain method) at the first Gauss point of the element 356 
bifurcation point, the relaxed potential of the small-strain domain approaches asymptotically a constant value, whereas the relaxed potential of the large-strain domain is active inside the shear band only and increases continuously with the prescribed displacement $v$. As a result, the relaxed stress approaches nearly a constant value.
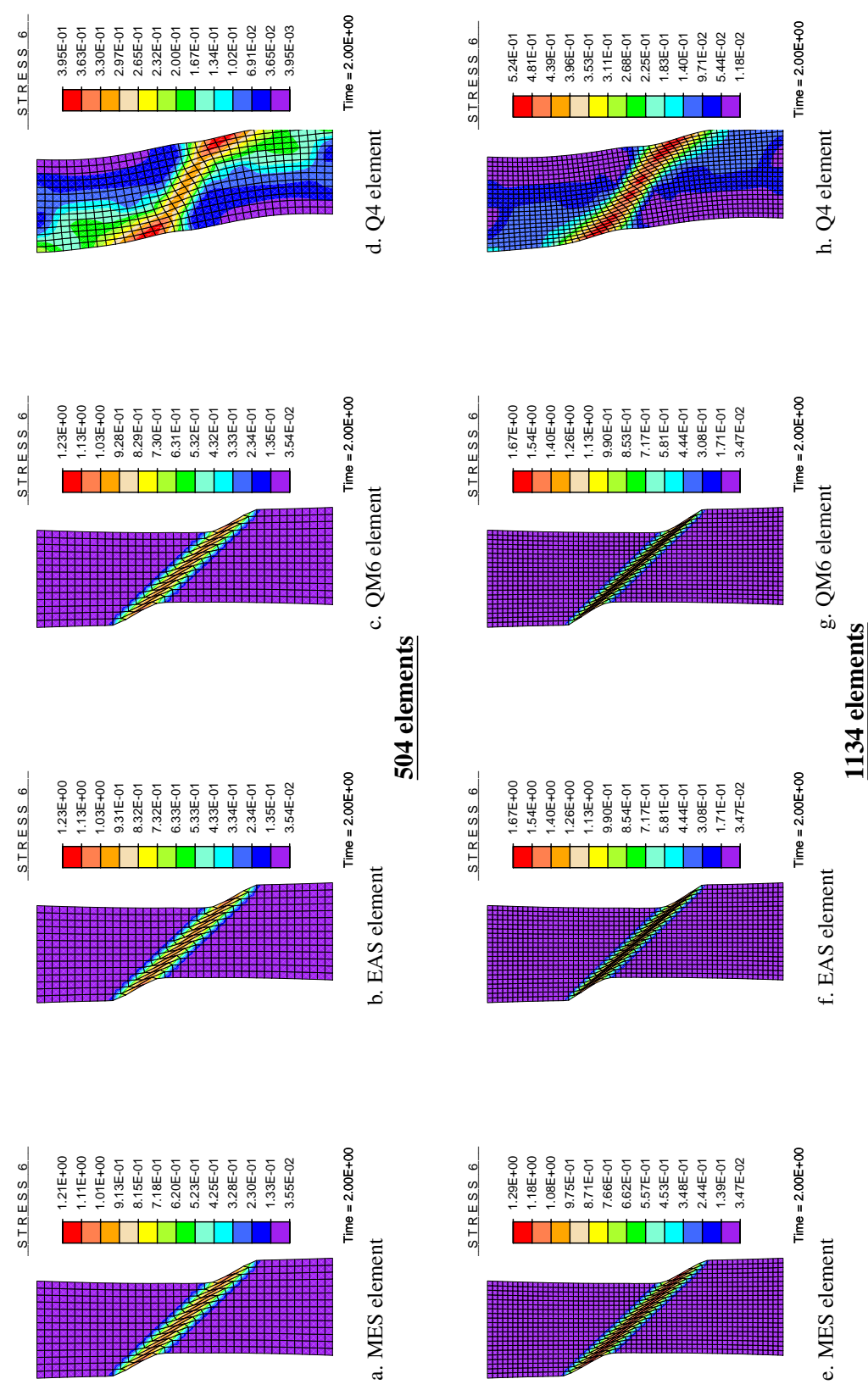

Fig. 7. Localization in tension. Comparison of the different finite element methods: Distribution of shear strain at $v=1.0 \mathrm{~mm}$ 
It is very interesting to see the performance of the different finite element formulations by considering the deformation shape of sample (Fig. 7). It is observed that the solution obtained by use of the Q4 element fails to capture effects of strain localization.

\section{CONCLUSIONS}

The paper focuses on a new approach for the treatment of strain localization in solid materials. The theory is based on minimization principles associated with micro-structure developments under the assumptions of a shear band of a zero thickness and the presence of a mixed potential inside the shear band.

Localization phenomena are regarded as micro-structure developments associated with nonconvex potentials. The nonconvexity of the mixed potential occurring due to the formation of strain localization is resolved by relaxation in order to ensure the wellposedness of the associated boundary value problem. The relaxed potential is obtained via local minimization problem of the mixed potential. The onset of localization is detected through the proposed optimization process. Numerical results show clearly meshindependent behavior in the sense that shear bands are as narrowly as the mesh-resolution allows, while all other features of the solution are independent of the chosen discretization.

\section{ACKNOWLEDGEMENT}

The research was supported through grants by the Vietnamese Government and Ministry of Education and Training as well as the Research School at Ruhr University Bochum, Germany.

\section{REFERENCES}

[1] Borja R., A finite element model for strain localization analysis of strongly discontinuous fields based on standard Galerkin approximation, Computer Methods in Applied Mechanics and Engineering, 190 (2000), 1529 - 1549.

[2] Lott-Crumpler D. A., The formation and properties of shear bands in viscoplastic materials, Modelling and Simulation in Materials Science and Engineering, 5 1997, 317 - 336.

[3] Hackl K., Hoppe U., On the calculation of microstructures for inelastic materials using relaxed enegies, Proceedings of IUTAM Symposium on Computational Mechanics of Solid Material at Large Strains, Miehe C.(ed.). Boston, (2001), 77 - 86.

[4] Ball J. M., James R. D., Fine phase mixtures as minimizer of energy, Archive for Rational Mechanics and Analysis, 100 (1987), 13 - 52.

[5] Dacorogna B., Direct methods in the calculus of variations, Springer-Verlag, Berlin, (1989).

[6] Kohn R., The relaxation of a double-well energy, Continuum Mechanics and Thermodynamics, 3 (1991), 193 - 236.

[7] Lambrecht C., Miehe C., Dettmar J., Energy relaxation of non-convex incremental stress potentials in a strain-softening elastic-plastic bar, International Journal of Solids and Structures, 40 (2003), 1369 - 1391.

[8] Bartel S., Carstensen C., Hackl K., Hoppe U., Effective relaxation for microstructure simulations: algorithms and applications, Computer Methods in Applied Mechanics and Engineering, 193 (2004), 5143 - 5175. 
[9] Hackl K., Heinen R., A micromechanical model for pretextured polycrystalline shape-memory alloys including elastic anisotropy, Continuum Mechanics and Thermodynamics, 19 (2008), $499-510$.

[10] Oda M. and Kamaza H. . Microstructure of shear bands and its relation to the mechanisms of dilatancy and failure of dense sranular soils, Géotechnique, 48 (1998), 465 - 481.

[11] Lewandowski J. J., Greer A. L., Temperature rise at shear bands in metallic glasses, Nature Materials, 5 (2006), 15 - 18.

[12] Simo J. C., Oliver J., Armero F., A analysis of strong discontinuities induced by strainsoftening in rate-independent inelastic solids, Computational Mechanics, 12 (1993), 277 - 296.

[13] Oliver J., Modelling strong discontinuities in solid mechanics via strain softening constitutive equations: Fundamental and Numerical Simulation, Monograph CIMNE No. 28, (1995).

[14] Finno R. J., Harris W. W., Mooney M. A., Viggiani G., Strain localization und undrained steady state of sand, Journal of Geotechnical Engineering, 122 (1996), 462 - 473.

[15] Taylor R. L., A finite element analysis program: User manual. http://www.ce.berkeley.edu/feap/, University of California at Berkeley, (2004).

[16] Kasper E. P., Taylor R. L., A mixed-enhanced strain method: Linear problems, No. UCB/SEMM-97/02, (1997).

[17] Simo J. C., Rifai M. S., A class of mixed assumed strain methods and the method of incompatible modes, International Journal for Numerical Methods in Engineering, 29 (1990), 1595 $-1638$.

[18] Taylor R. L., Beresford P. J., Wilson E. L., A non-conforming element for stress analysis, International Journal for Numerical Methods in Engineering, 10 (1976), 1211 - 1219.

[19] Zienkiewicz O. C., Taylor R. L., The finite element method. Volume 1: The Basis (5nd edn), Butterworth-Heinmann: London, (2000). 